Article

\title{
Unveiling the Different Reactivity of Bent and Linear Three-Atom-Components Participating in [3 + 2] Cycloaddition Reactions
}

\author{
Mar Ríos-Gutiérrez ${ }^{1, *(D)}$, Luis R. Domingo ${ }^{1}$ (D) and Fatemeh Ghodsi ${ }^{2}$ \\ 1 Department of Organic Chemistry, University of Valencia, Dr Moliner 50, Burjassot, 46100 Valencia, Spain; \\ domingo@utopia.uv.es \\ 2 Department of Chemistry, University of Sistan and Baluchestan, Zahedan 98135-674, Iran; \\ ghodsi93@pgs.usb.ac.ir \\ * Correspondence: rios@utopia.uv.es
}

Citation: Ríos-Gutiérrez, M.;

Domingo, L.R.; Ghodsi, F. Unveiling the Different Reactivity of Bent and Linear Three-Atom-Components Participating in [3+2] Cycloaddition Reactions. Organics 2021, 2, 274-286. https://doi.org/10.3390/org2030014

Academic Editors: David StC Black and Tomasz K. Olszewski

Received: 17 May 2021

Accepted: 9 August 2021

Published: 13 August 2021

Publisher's Note: MDPI stays neutral with regard to jurisdictional claims in published maps and institutional affiliations.

Copyright: (c) 2021 by the authors. Licensee MDPI, Basel, Switzerland. This article is an open access article distributed under the terms and conditions of the Creative Commons Attribution (CC BY) license (https:// creativecommons.org/licenses/by/ $4.0 /)$.

\begin{abstract}
The reactivity of a series of pairs of bent and linear three-atom-component (B-TACs and L-TACs) participating in [3 + 2] cycloaddition (32CA) reactions towards ethylene and electrophilic dicyanoethylene (DCE) have been studied within the Molecular Electron Density Theory. While the pseudodiradical structure of B-TACs changes to that of pseudoradical or carbenoid L-TACs upon dehydrogenation, zwitterionic B-TACs remain unchanged. Conceptual Density Functional Theory (CDFT) indices characterize five of the nine TACs as strong nucleophiles participating in polar reactions towards electrophilic ethylenes. The activation energies of the 32CA reactions with electrophilic DCE range from 0.5 to $22.0 \mathrm{kcal} \cdot \mathrm{mol}^{-1}$, being between 4.3 and $9.1 \mathrm{kcal} \cdot \mathrm{mol}^{-1}$ lower than those with ethylene. In general, B-TACs are more reactive than their L-TAC counterparts. A change in the regioselectivity is found in these polar 32CA reactions; in general, while B-TACs are meta regioselective, L-TACs are ortho regioselective. The geometrical parameters of the transition state structures suggest that the formation of the single bond involving the most electrophilic carbon of DCE is more advanced. A change in the asynchronicity in the reactions involving B-TACs and L-TACs is also found.
\end{abstract}

Keywords: $[3+2]$ cycloaddition reactions; bent and linear three-atom-components; reactivity; regioselectivity; molecular electron density theory

\section{Introduction}

Cycloaddition reactions are one of the most useful tools in organic synthesis as they permit to obtain cyclic compounds with a regio- and/or stereoselective fashion [1,2]. [3 + 2] cycloaddition (32CA) reactions, which involve the 1,3-addition of an ethylene to a three-atom-component (TAC) (see Scheme 1), are an important type of cycloaddition allowing the formation of five-membered heterocycles of great pharmaceutical and industrial interest $[2,3]$. \begin{tabular}{c}
$Z$ \\
\hdashline$:$ \\
\hdashline $4 e$ \\
$\ddots$ \\
$X$
\end{tabular}

TAC

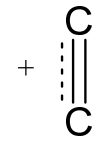

multiple bond system
$32 \mathrm{CA}$

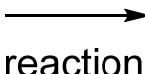<smiles>[Y]1[c]cc[c]1</smiles>

heterocyclic compound
Scheme 1. 32CA reaction between a TAC and an ethylene derivative.

Recent Molecular Electron Density Theory [4] (MEDT) studies of 32CA reactions have allowed establishing a very good correlation between the electronic structure of 
TACs and their reactivity [5]. The 32CA reactions of pseudodiradical, pseudo(mono)radical, carbenoid and zwitterionic TACs have been classified into pseudodiradical-type (pdr-type), pseudo(mono)radical-type (pmr-type), carbenoid-type (cb-type) and zwitterionic-type (zw-type) reactions (see Figure 1) [5]; thus, while pdr-type 32CA reactions take place very easily, $z w$-type 32CA reactions need a nucleophilic/electrophilic activation (see Figure 1).

\begin{tabular}{lll}
\hline $\begin{array}{c}\text { Azomethine } \\
\text { ylide }\end{array}$ & $\begin{array}{c}\text { Szomethine } \\
\text { imine }\end{array}$ & zwitterionic \\
\hline pseudodiradical & pseudoradical & carbenoid \\
\hline pdr-type & Reactivity
\end{tabular}

Figure 1. The four representative reactivity models in 32CA reactions.

Organic reactions are classified as non-polar and polar reactions, the latter being favored with the increase in the polar character of the reaction [6]. The analysis of the global electron density transfer (GEDT) $[7,8]$ at the transition state structures (TSs) allows characterizing the polar character of a reaction; while GEDT values below 0.05 e characterize non-polar processes, values above 0.20 e characterize polar processes. Very recently, cycloadditions have been classified as forward electron density flux (FEDF) and reverse electron density flux (REDF) reactions, depending on the direction of the electron density flux at the TS [9]. Reactions with the direction of the electron density flux from the diene or TAC to the ethylene are classified as FEDF. Non-polar reactions are classified as null electron density flux (NEDF) [10]. This classification is unequivocal, as the GEDT is a measure of the actual electron density transfer at the TSs. Thus, the DA reaction between butadiene and ethylene, classified as "normal electron demand" [11] within Sustmann's classification [12], is non-polar as the GEDT at the TS is negligible, with 0.0 e [13], being classified as NEDF.

TACs can also be classified, depending on their geometrical structure, into bent TACs (B-TACs), such as azomethine ylide, and linear TACs (L-TACs), such as nitrile ylide (see Figure 1). In many cases, L-TACs can be seen as resulting from the dehydrogenation of B-TACs (see Scheme 2). As a result, the central $Y$ atom of the B-TAC changes from a trigonal arrangement at the B-TAC to a linear one at the L-TAC, thus justifying the change in the geometry of the TACs.

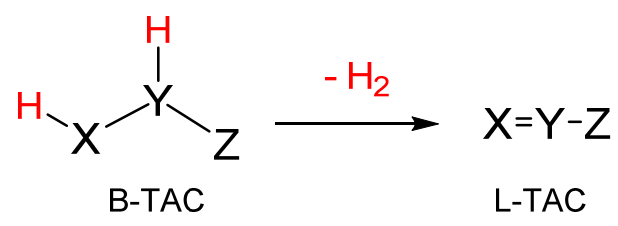

Scheme 2. Chemical relationship between some B- and L-TACs.

In general, B-TACs are more reactive and regioselective towards electrophilic ethylenes than L-TACs. In order to establish some general correlations between the electronic structure and reactivity of B- and L-TACs, an MEDT study of the 32CA reactions of five pairs of B-TACs 1-4 and L-TACs 5-8 (see Table 1) with ethylene 9 and the electrophilic dicyanoethylene (DCE) 10 is herein performed. 
Table 1. Atom composition and name of the pairs of B- and L-TACs studied herein.

\begin{tabular}{cccccc}
\hline \multicolumn{3}{c}{ B-TACs } & \multicolumn{2}{c}{ L-TACs } \\
\hline \multicolumn{1}{c}{$\mathbf{X 1 - N 2 - Z 3}$} & Name & & $\mathbf{X 1}-\mathbf{N 2}-\mathbf{Z 3}$ & Name \\
\hline $\mathbf{1}$ & $\mathrm{H}_{2} \mathrm{C}-\mathrm{NH}-\mathrm{CH}_{2}$ & azomethine ylide & $\mathbf{5}$ & $\mathrm{H}_{2} \mathrm{C}-\mathrm{N}-\mathrm{CH}$ & nitrile ylide \\
$\mathbf{2}$ & $\mathrm{H}_{2} \mathrm{C}-\mathrm{NH}-\mathrm{NH}$ & azomethine imine & $\mathbf{6}^{\prime}$ & $\mathrm{H}_{2} \mathrm{C}-\mathrm{N}-\mathrm{N}$ & diazomethane \\
& & & $\mathbf{6}^{\prime \prime}$ & $\mathrm{HC}-\mathrm{N}-\mathrm{NH}$ & nitrile imine \\
$\mathbf{3}$ & $\mathrm{NH}-\mathrm{NH}-\mathrm{NH}$ & azimine & 7 & $\mathrm{NH}-\mathrm{N}-\mathrm{N}$ & azide \\
$\mathbf{4}$ & $\mathrm{H}_{2} \mathrm{C}-\mathrm{NH}-\mathrm{O}$ & nitrone & $\mathbf{8}$ & $\mathrm{HC}-\mathrm{N}-\mathrm{O}$ & nitrile oxide \\
\hline
\end{tabular}

\section{Computational Methods}

All stationary points were optimized using the MPWB1K functional [14], together with the 6-311G(d,p) basis set [15]. The optimizations were carried out using the Berny analytical gradient optimization method $[16,17]$. The stationary points were characterized by frequency computations in order to verify that TSs have one and only one imaginary frequency. The intrinsic reaction coordinate (IRC) paths [18] were traced in the gas phase in order to check and obtain the energy profiles connecting each TS to the two associated minima of the proposed mechanism, i.e., reactants and products, using the second-order González-Schlegel integration method [19,20]. The GEDT [7] was computed by the sum of the atomic charges $(\mathrm{q})$ of the atoms belonging to each framework at the TSs; GEDT $=\Sigma q_{\mathrm{f}}$.

The electronic structures of the reagents were characterized by the topological analysis of the electron localisation function [21] (ELF) and by a Natural Population Analysis (NPA) [22,23]. CDFT reactivity indices [24,25] were computed at the B3LYP/6-31G(d) level since original reactivity scales were established at that method, using the equations given in reference [25]. The global electrophilicity index [26] $\omega$ is given by the following expression, $\omega=\left(\mu^{2} / 2 \eta\right)$, in terms of the electronic chemical potential [24] $\mu$ and the chemical hardness [27] $\eta$. Both quantities may be approached in terms of the one-electron energies of the frontier molecular orbitals HOMO and LUMO, $\varepsilon_{\mathrm{H}}$ and $\varepsilon_{\mathrm{L}}$, as $\mu \approx\left(\varepsilon_{\mathrm{H}}+\varepsilon_{\mathrm{L}}\right) / 2$ and $\eta \approx\left(\varepsilon_{\mathrm{L}}-\varepsilon_{\mathrm{H}}\right)$, respectively. The global nucleophilicity index, [28] $N$, based on the HOMO energies obtained within the Kohn-Sham scheme [29], is defined as $N=\mathrm{E}_{\mathrm{HOMO}}(\mathrm{Nu})-\mathrm{E}_{\mathrm{HOMO}}(\mathrm{TCE})$, where tetracyanoethylene (TCE) is the reference. Electrophilic $P_{k}^{+}$and nucleophilic $P_{k}^{-}$Parr functions [30] were obtained through the analysis of the Mulliken atomic spin densities (ASD) of the radical anion of ethylene $\mathbf{9}$ and DCE 10 and the radical cations of TACs 1-8.

All computations were carried out with the Gaussian 16 suite of programs [31]. ELF studies were performed with the TopMod program [32], using the corresponding MPWB1K/6-311G(d,p) monodeterminantal wavefunctions and considering the standard cubical grid of step size of 0.1 Bohr. The molecular geometries and ELF basin attractor positions were visualized using the GaussView program [33].

\section{Results and Discussion}

This MEDT study is divided into three parts: (i) first, the electronic structures of BTACs and L-TACs based on ELF analysis and NPA are compared; (ii) then, the participation of B-TACs and L-TACs in polar 32CA reactions is analyzed within CDFT; and finally, (iii) the 32CA reactions of the nine TACs 1-8 with ethylene 9 and DCE 10 are explored in order to compare the reactivity of B-TACs and L-TACs in non-polar and polar processes.

\subsection{Comparison of the Electronic Structures of B-TACs and L-TACs}

The electron density distribution of matter determines both its physical and chemical properties, i.e., reactivity [4]. The topological analysis of the ELF has proven to be a powerful tool to establish a straightforward connection between the electron density distribution and the chemical structure [21]. Hence, ELF analysis and NPA [22,23] of the nine selected TACs were carried out in order to characterize the structural changes between 
the B-TAC/L-TAC pairs. ELF basin attractor positions and populations of the valence basins are given in Figure 2.
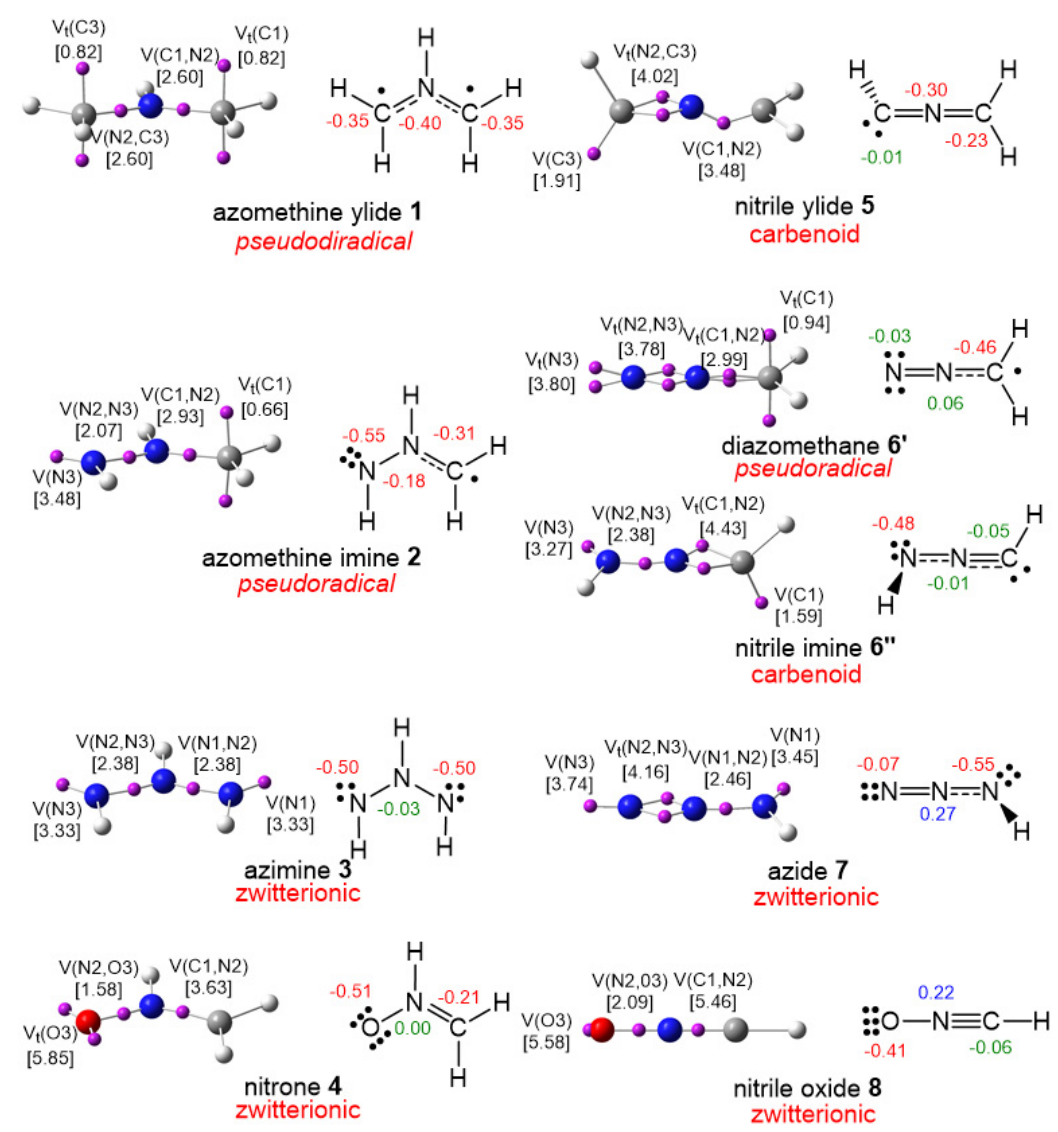

Figure 2. ELF basin attractor positions, ELF-based Lewis-like structures and TAC types, together with calculated natural atomic charges, in number of electrons e, of TACs 1-8. Negative charges are colored in red, positive charges in blue and negligible charges in green.

The presence of two carbon pseudoradical centers (see the two $\mathrm{V}_{\mathrm{t}}(\mathrm{C} 1[3])$ monosynaptic basins integrating a total of 0.82 e each one in Figure 2) and two overpopulated C1[3]-N2 single bonds characterize azomethine ylide 1 as a pseudodiradical B-TAC. The presence of the two pseudoradical centers accounts for the high reactivity of this TAC. After dehydrogenation of B-TAC $\mathbf{1}$, the electron density of nitrile ylide 5 is reorganized into a carbenoid allenic linear structure characterized by the presence of a non-bonding electron density at the C3 carbon integrating 1.91 e (see the V(C3) monosynaptic basin in Figure 2) and two nonconjugated C1[3]-N2 double bonds. Thus, nitrile ylide 5 is characterized as a carbenoid L-TAC.

The presence of a pseudoradical $\mathrm{C} 1$ carbon (see the two $\mathrm{V}(\mathrm{C} 1)$ monosynaptic basins, integrating a total of 0.66 e in Figure 2), a C1-N2-overpopulated single bond and an $\mathrm{N} 2-\mathrm{N} 3$ single bond characterize azomethine imine 2 as a pseudo(mono)radical allylic BTAC. Two different L-TACs can be found after dehydrogenation of $\mathbf{2}$, depending on which of the $\mathrm{C} 1-\mathrm{N} 2$ or $\mathrm{N} 2-\mathrm{N} 3$ bonds is involved; i.e., diazomethane $\mathbf{6}^{\prime}$ and nitrile imine $\mathbf{6}^{\prime \prime}$, respectively. At diazomethane $6^{\prime}$, the electron density is mainly redistributed into the $\mathrm{N} 2-\mathrm{N} 3$ bond, so this L-TAC has a rather allenic, but still pseudo(mono)radical, structure (see the two $\mathrm{V}(\mathrm{C} 1)$ monosynaptic basins integrating a total of $0.94 \mathrm{e}$ in Figure 2). On the other hand, at nitrile imine $\mathbf{6}^{\prime \prime}$ the electron density is distributed between the $\mathrm{C} 1-\mathrm{N} 2$ region and the $\mathrm{C} 1$ carbon, thus presenting a carbenoid L-TAC structure, with a non-bonding electron density of 1.59 e at the carbenoid $\mathrm{C} 1$ carbon (see the $\mathrm{V}(\mathrm{C} 1)$ monosynaptic basin in Figure 2), and an N2-C3 double bond. 
The electron density of B-TAC azimine 3 is shared between the two N1[3]-N2 single bonds, integrating $2.38 \mathrm{e}$, and two terminal N1[3] nitrogens presenting a non-bonding electron density of 3.33 e in each one. Azimine 3 is characterized as zwitterionic TAC, due to the absence of any pseudoradical or carbenoid center, but it does not present any multiple bond. After dehydrogenation of $\mathbf{3}$, the electron density of azide 7 is mainly redistributed into the N2-N3 bonding region, which becomes a double bond; thus, azide 7 remains as a zwitterionic L-TAC.

Finally, nitrone 4 is characterized as a zwitterionic allylic B-TAC due to the absence of any pseudoradical or carbenoid center, but it has an N2-C3 double bond. After dehydrogenation of 4 , the $\mathrm{N} 2-\mathrm{C} 3$ bonding region in nitrile oxide 8 becomes a triple bond, but it remains as a zwitterionic structure. It can be inferred from this that the 2 e freed upon dehydrogenation of 4 have almost entirely been redistributed into the N2-C3 region.

This ELF analysis shows that while some pairs of B-TACs and L-TACs, such as zwitterionic $4 / 8$ and $3 / 7$, and pseudo (mono)radical $2 / 6^{\prime}$, retain the same structural type, other TACs, such as pseudodiradical $\mathbf{1}$ vs. carbenoid 5, and pseudo(mono)radical $\mathbf{2}$ vs. carbenoid $\mathbf{6}^{\prime \prime}$, change their structure.

NPA of the $\mathrm{X} 1-\mathrm{N} 2-\mathrm{Y} 3$ framework of the nine TACs shows that the charges of the B-TACs range from -1.10 e (1) to -0.72 e (4), while those of the L-TACs range from -0.55 e (5) to -0.36 e (7), emphasizing that their electronic structure cannot be represented by a set of resonance Lewis structures [5], but they are the result of the distribution of the whole molecular electron density depending on the nature and relative positions of the nuclei forming the TAC. Thus, as the nuclei are more electronegative than those of the hydrogen, the $\mathrm{X} 1-\mathrm{N} 2-\mathrm{Y} 3$ frameworks are negatively charged; the ones of B-TACs are more negatively charged as they have more hydrogens. Thus, while azomethine ylide $\mathbf{1}$ is the TAC with the most negatively charged core framework, $-1.10 \mathrm{e}$, as it has five hydrogens, nitrile oxide 8 is the TAC with the least negatively charged core structure, $-0.25 \mathrm{e}$, as it has only one hydrogen. Consequently, the traditional definition of TACs as 1,3-dipoles mainly described by 1,2-zwitterionic Lewis structures [34] should be completely avoided [5].

\subsection{Analysis of CDFT Reactivity Indices at the Reagents}

The reactivity indices defined within CDFT [24,25] have become a powerful tool to study chemical reactivity. Thus, in order to understand the different reactivity of B- and L-TACs, the global CDFT indices, namely, the electronic chemical potential $\mu$, the chemical hardness, $\eta$, the electrophilicity, $\omega$, and the nucleophilicity, $N$, at TACs 1-8, ethylene 9 and DCE 10, were studied (see Table 2). They were computed at the B3LYP/6-31G(d) computational level, as it was used to establish the electrophilicity and nucleophilicity scales [25].

Table 2. B3LYP/6-31G(d) global electronic chemical potential, $\mu$, chemical hardness, $\eta$, electrophilicity, $\omega$, and nucleophilicity, $N$, in eV, of simplest TACs 1-8, ethylene 9 and DCE 10.

\begin{tabular}{lcccc}
\hline Structure & $\boldsymbol{\mu}$ & $\boldsymbol{\eta}$ & $\boldsymbol{\omega}$ & $\boldsymbol{N}$ \\
\hline TAC 1 & -1.81 & 4.47 & 0.37 & 5.07 \\
TAC 2 & -2.70 & 5.02 & 0.72 & 3.92 \\
TAC 3 & -3.70 & 5.62 & 1.22 & 2.61 \\
TAC 4 & -3.43 & 5.54 & 1.06 & 2.92 \\
TAC 5 & -2.90 & 5.45 & 0.77 & 3.50 \\
TAC 6 & -3.64 & 4.73 & 1.40 & 3.11 \\
TAC 6 & -3.54 & 5.87 & 1.07 & 2.64 \\
TAC 7 & -4.24 & 6.54 & 1.37 & 1.62 \\
TAC 8 & -3.40 & 7.94 & 0.73 & 1.75 \\
Ethylene 9 & -3.37 & 7.77 & 0.73 & 1.87 \\
DCE 10 & -5.64 & 5.65 & 2.82 & 0.65 \\
\hline
\end{tabular}


Most of the electronic chemical potentials [24] $\mu$ of the TACs, which range from -1.81 (azomethine ylide 1 ) to -4.24 (azide 7) $\mathrm{eV}$, are close to that of ethylene $9,-3.37 \mathrm{eV}$, indicating that the corresponding 32CA reactions will have non-polar character. On the other hand, these values are higher than electronic chemical potentials $\mu$ of DCE 10, $-5.64 \mathrm{eV}$, indicating that the GEDT will flux from the TACs towards DCE 10, the reactions being classified as FEDF [9]. The values of the electronic chemical potentials $\mu$ of these TACs decrease with the increase in the number of electronegative atoms present in the TAC, B-TACs presenting higher values than L-TACs.

The chemical hardness [27] $\eta$ of the TACs ranges from 4.47 (azomethine ylide 1) to 7.92 (nitrile oxide 8) eV. A similar increase in the chemical hardnesses $\eta$ is observed. Except diazomethane $\mathbf{6}^{\prime}$, L-TACs are harder species than B-TACs, suggesting more resistance of the former against perturbations. A good correlation between the chemical hardness and the electronic structure of the TAC is observed; the chemical hardness increases in the order pseudodiradical < pseudo (mono)radical < carbenoid < zwitterionic.

The electrophilicity $\omega$ indices [26] of the TACs, ranging from 0.37 (azomethine ylide 1) to 1.40 (diazomethane $\mathbf{6}^{\prime}$ ) eV, permit to classify TACs $3, \mathbf{4}, \mathbf{6}^{\prime}, \mathbf{6}^{\prime \prime}$ and $\mathbf{7}$ as moderate electrophiles, and TACs 1, 2, 5 and $\mathbf{8}$ as marginal electrophiles [25]; therefore, none of them will participate in polar reactions towards a nucleophilic ethylene. On the other hand, the nucleophilicity $N$ indices [28] of the TACs, ranging from 1.62 (azide 7) to 5.07 (azomethine ylide 1) eV, makes that, excluding L-TACs azide 7 and nitrile oxide 8 , they are classified as moderate or strong nucleophiles. Azomethine ylide 1, with $N>4.0 \mathrm{eV}$, is classified as a supernucleophile [35]. Consequently, TACs $\mathbf{1}, \mathbf{2}, \mathbf{5}$ and $\mathbf{6}^{\prime}$ will participate in polar 32CA reactions towards electrophilic ethylenes. In general, B-TACs are more nucleophilic than their L-TAC counterparts.

Ethylene 9, a marginal electrophile with a $\omega=0.73 \mathrm{eV}$ and a marginal nucleophile with a $N=1.87 \mathrm{eV}$, cannot participate in polar reactions, while DCE 10, a strong electrophile with a $\omega=2.82 \mathrm{eV}$, participates in polar 32CA reactions; the reactions of DCE 10 with B-TACs $\mathbf{1}$ and $\mathbf{2}$, and L-TACs 5 and $\mathbf{6}^{\prime}$ will have a highly polar character, in clear agreement with the GEDT values computed at the more favorable TSs (see Section 3.3).

The most favourable two-center electrophilic/nucleophilic interaction defines regioselectivity in polar reactions [36]. The analysis of electrophilic $P_{k}^{+}$and nucleophilic $P_{k}^{-}$ Parr functions [30], derived from the excess of spin electron density reached via the GEDT process, has proven to be the most accurate and insightful tool for the study of the local reactivity in polar and ionic processes. Therefore, the nucleophilic $P_{k}^{-}$Parr functions of TACs 1-8 and the electrophilic $P_{k}^{+}$Parr functions of DCE 10 were analyzed in order to know the different local reactivity of B-TACs and L-TACs (see Figure 3).

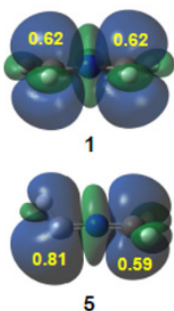

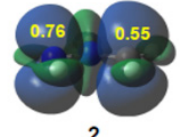
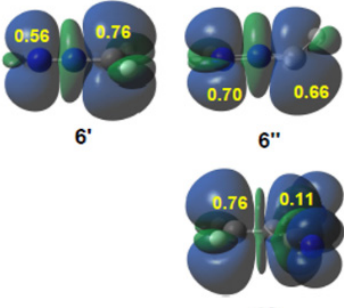

10
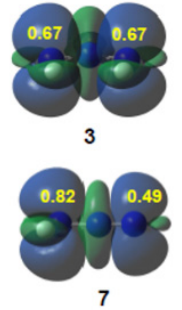

7

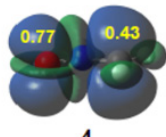

4

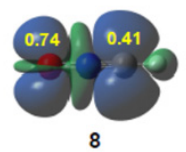

Figure 3. 3D representation of the Mulliken atomic spin density maps of radical cations (1-8) ${ }^{\cdot+}$ and radical anion $\mathbf{1 0}^{-}$- together with the nucleophilic $P_{k}^{-}$Parr functions of TACs 1-8 and the electrophilic $P_{k}^{+}$Parr functions of DCE 10.

When the changes in the Parr functions were analyzed by pairs of B-TACs and L-TACs, no noticeable trend could be found. In non-symmetric B-TACs 2 and 4 and L-TACs 5, 6" 
and 8, the more electronegative heteroatom holds the higher nucleophilic $P_{k}^{-}$Parr function (see Figure 3).

The most nucleophilic center in L-TACs is the non-dehydrogenated center, except in nitrile ylide 5, where the carbenoid $\mathrm{CH}$ carbon is more nucleophilic than the methylene $\mathrm{CH}_{2}$ one. An opposite regioselectivity is expected only in pair azomethine imine 2/diazomethane $6^{\prime}$.

3.3. Study of the Reaction Paths Associated with the 32CA Reactions of TACs 1-8 with Ethylene $\mathbf{9}$ and with the Electrophilic DCE 10

3.3.1. Study of the 32CA Reactions with Ethylene 9

First, the non-polar 32CA reactions of TACs 1-8 with ethylene 9 were investigated. Ethylene 9, a marginal electrophile and marginal nucleophile, does not participate in polar reactions (see Table 1). Only one reaction path is feasible for the nine 32CA reactions, which take place through a one-step mechanism (see Scheme 3). The gas-phase relative energies of the stationary points are given in Table 2, while the total electronic energies are reported in Table S1 in Supplementary Material.
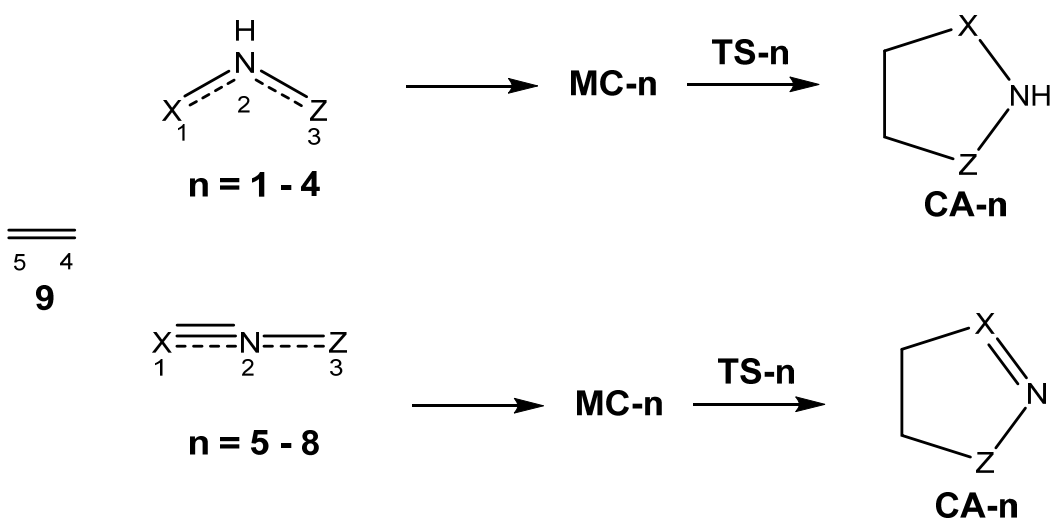

Scheme 3. Simplest 32CA reactions of TACs 1-8 with ethylene 9.

A series of molecular complexes (MCs) in an early stage of the reactions in which both reagents are weakly bound by non-covalent interactions can be located. They are found between $0.8\left(\mathbf{M C}-6^{\prime \prime}\right)$ and $3.3(\mathbf{M C}-4) \mathrm{kcal} \cdot \mathrm{mol}^{-1}$ below the separated reagents (see Scheme 3 and Table 3). The relative energies of the TSs of the 32CA reactions of B-TACs 1-4 with respect to the separated reagents are found between 0.1 (TS-1) and 15.5 (TS-3) $\mathrm{kcal} \cdot \mathrm{mol}^{-1}$, while those associated with L-TACs 5-8 are found between 7.2 (TS-5) and 21.1 (TS-7) $\mathrm{kcal} \cdot \mathrm{mol}^{-1}$ (see Table 3); the reactions are strongly exothermic by between $81.4(\mathbf{C A}-5)$ to $-30.0(\mathbf{C A}-7) \mathrm{kcal} \cdot \mathrm{mol}^{-1}$. The relative energy difference between the TSs associated with the 32CA reactions of each pair of B- and L-TACs are: 7.1 ( $p d r$ 1/cb 5), 9.9 $\left(p m r 2 / p m r 6^{\prime}\right), 1.6\left(p m r 2 / c b 6^{\prime \prime}\right), 5.6(z w 3 / z w 7)$ and $3.8(z w 4 / z w 8) \mathrm{kcal} \cdot \mathrm{mol}^{-1}$.

Table 3. MPWB1K/6-311G(d,p) gas phase relative electronic energies, in $\mathrm{kcal} \cdot \mathrm{mol}^{-1}$, with respect to the separated reagents, of the stationary points involved in the 32CA reactions of TACs 1-8 with ethylene 9 .

\begin{tabular}{lccc}
\hline TAC & MC-n & TS-n & CA-n \\
\hline $\mathbf{1}$ & -1.8 & 0.1 & -77.8 \\
$\mathbf{2}$ & -2.3 & 6.4 & -59.4 \\
$\mathbf{3}$ & -2.9 & 15.5 & -43.2 \\
$\mathbf{4}$ & -3.3 & 11.8 & -41.0 \\
\hline $\mathbf{5}$ & -1.2 & 7.2 & -81.5 \\
$\mathbf{6}^{\prime}$ & -1.0 & 16.3 & -42.6 \\
$\mathbf{6}^{\prime \prime}$ & -0.8 & 8.0 & -68.9 \\
$\mathbf{7}$ & -3.1 & 21.1 & -30.0 \\
$\mathbf{8}$ & -1.5 & 15.6 & -50.8 \\
\hline
\end{tabular}


Some interesting conclusions can be drawn from the relative energies: (i) the TSs of the L-TACs are by between $1.6\left(6^{\prime \prime}\right)$ and $9.9\left(6^{\prime}\right) \mathrm{kcal} \cdot \mathrm{mol}^{-1}$ above in energy than those of the B-TACs; (ii) as expected, the reactivity of B-TACs follows the trend $p d r>p m r>z w$ [5], while that of L-TACs decreases in the order $c b>z w>p m r$, the reactivity of diazomethane $\mathbf{6}^{\prime}$ being an exception; and (iii) except pseudo(mono)radical $\mathbf{6}^{\prime}$, which presents a high activation energy of $16.3 \mathrm{kcal} \cdot \mathrm{mol}^{-1}$, only zwitterionic TACs present a low reactivity as the activation energies are found higher than $11.8 \mathrm{kcal} \cdot \mathrm{mol}^{-1}$ (nitrone 4).

The gas-phase optimized TS geometries are shown in Figure 4, together with the distances between the four interacting centers. Some interesting information is obtained from these distances: (i) except TS-1 and TS-3, which are completely synchronous, the other seven TSs present a low asynchronicity measured by $\Delta \mathrm{d}$, which varies from $0.02 \AA$ at TS-7 to $0.14 \AA$ at TS-8; (ii) from a geometrical point of view, TS-8, TS-6' and TS-6" ${ }^{\prime \prime}$, associated with L-TACs $8, \mathbf{6}^{\prime}$ and $\mathbf{6}^{\prime \prime}$, are more asynchronous than TS-4 and TS-2, associated with their B-TAC counterparts 4 and 2 . The TSs associated with L-TACs are in general more asynchronous than those associated with B-TACs; (iii) five of the nine TSs agrees with Hammond's principle [37]; however, Hammond's correlation between distances and reactivity does not hold for the TSs of L-TACs $6^{\prime}$ and 8, and B-TACs 3 and 4; and finally, (iv) excluding TS- 6 " and TS-8, the most nucleophilic center of the TAC participates in the shorter two-center interaction in non-symmetric TSs.

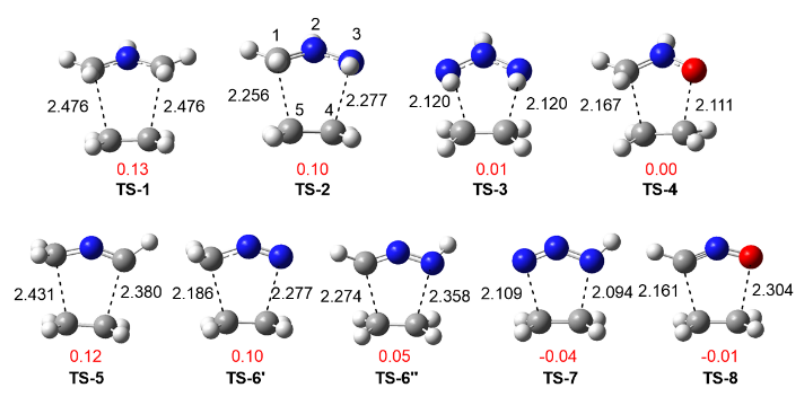

Figure 4. MPWB1K/6-311G(d,p) gas-phase optimized geometries of the TSs involved in the 32CA reactions of TACs 1-8 with ethylene 9. Distances are given in angstroms, $\AA$, while the GEDT values computed at the TAC framework, in red, are given in number of electrons, e.

The polar character of the nine 32CA reactions involving ethylene 9 was checked by computing the GEDT at every TS (see Figure 4). The analysis of the GEDT values shown in Figure 4 indicates that five of the nine reactions are non-polar, with GEDT values lower than 0.05 e, while four reactions show a low polar character, with GEDT values between 0.10 and $0.13 \mathrm{e}$. It is worth mentioning that these values over $0.10 \mathrm{e}$ are not the consequence of favorable nucleophilic/electrophilic interactions. As expected, these GEDT values confirm the general low-polar character of 32CA reactions involving the poor electrophilic ethylene 9. The non-polar reactions correspond to those involving B-TACs 3 and 4 , and L-TACs 8 and $\mathbf{6}^{\prime \prime}$, with GEDT values ca. 0 e. When the GEDT values were compared by pairs of TSs of B-TACs and L-TACs, no substantial changes were observed. Only TS- $6^{\prime \prime}$, involving L-TAC nitrile imine $6^{\prime \prime}$, is notably less polar than TS-2, involving its B-TAC partner azomethine imine 2.

\subsubsection{Study of the $32 \mathrm{CA}$ Reactions with Electrophilic DCE 10}

Except for symmetric B-TACs 1 and 3, the 32CA reactions of these TACs with DCE 10 can take place along two competitive regioisomeric paths, named meta and ortho (see Scheme 4). While the ortho reaction paths involve the formation of the Z3-C4 single bond, the meta ones involve the formation of the $\mathrm{X} 1-\mathrm{C} 4$ one. In every TAC, Z3 labels the most electronegative atom. These 32CA reactions take place through a one-step mechanism (see Scheme 4). The gas-phase relative energies of the stationary points are given in Table 4, while the total electronic energies are reported in Table S2 in Supplementary Material. 


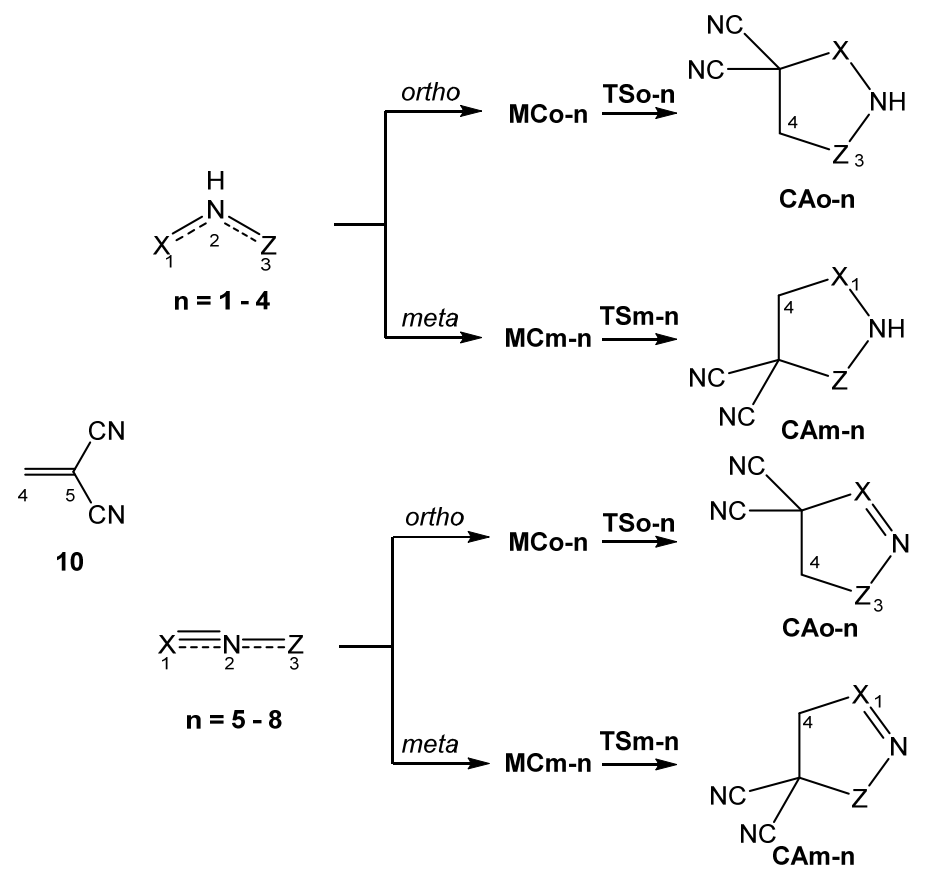

Scheme 4. Regioisomeric reaction paths associated with the 32CA reactions between TACs 1-8 and DCE 10.

Table 4. MPWB1K/6-311G(d,p) gas phase relative electronic energies, in $\mathrm{kcal} \cdot \mathrm{mol}^{-1}$, with respect to the separated reagents, of the stationary points involved in the 32CA reactions of TACs 1-8 with DCE 10

\begin{tabular}{lcccccc}
\hline \multicolumn{1}{c}{ TAC } & MCo-n & TSo-n & CAo-n & MCm-n & TSm-n & CAm-n \\
\hline $\mathbf{1} p d r$ & - & - & -76.5 & & & \\
$\mathbf{2} p m r$ & -8.3 & -7.4 & -49.6 & -5.9 & -4.6 & -53.6 \\
$\mathbf{3} z w$ & -4.0 & 5.9 & -36.9 & & & \\
$\mathbf{4} z w$ & -7.8 & -0.3 & -36.5 & -9.0 & 5.1 & -31.8 \\
\hline $\mathbf{5} c b$ & -6.9 & -6.3 & -76.1 & -4.2 & -1.8 & -73.8 \\
$\mathbf{6}^{\prime} p m r$ & -4.0 & 11.3 & -34.2 & -2.6 & 4.6 & -31.2 \\
$\mathbf{6}^{\prime \prime} c b$ & -5.2 & 1.0 & -60.9 & -4.8 & 0.2 & -61.1 \\
$\mathbf{7} z w$ & -2.5 & 21.9 & -19.0 & -5.6 & 16.4 & -18.6 \\
$\mathbf{8} z w$ & -4.0 & 15.3 & -38.3 & -1.5 & 13.6 & -38.3 \\
\hline
\end{tabular}

A series of MCs can be found in an early stage of the reactions, being located between $2.5(\mathbf{M C m}-7)$ and $9.0(\mathbf{M C o}-4) \mathrm{kcal} \cdot \mathrm{mol}^{-1}$ below the separated reagents (see Scheme 4 and Table 4). The relative energies of the more favorable regioisomeric TSs range between -7.4 (TSm-2) and 5.9 (TSm-3) kcal- $\mathrm{mol}^{-1}$ for B-TACs 1-4 and between -6.3 (TSm-5) and $21.9(\mathrm{TSm}-7) \mathrm{kcal} \cdot \mathrm{mol}^{-1}$ for L-TACs $5-8$, the reactions being exothermic by between $18.6(\mathbf{C A o}-7)$ and $76.6(\mathbf{C A m}-\mathbf{1}) \mathrm{kcal} \cdot \mathrm{mol}^{-1}$. These data show the highly variable reactivity among TACs.

Some interesting conclusions can be drawn from the relative energies: (i) five of the fifteen TSs are located below the separated reagents. However, if the formation of the corresponding MCs is considered, the activation energies become positive; (ii) the high reactivity of supernucleophilic azomethyne ylide 1 makes the 32CA reaction towards electrophilic DCE 10 barrierless; neither MCo-1 nor TSo-1 were found on the PES; (iii) the activation energies, with respect to the corresponding MCs, range from 0.6 (TSm-5) to 22.0 (TSo-7) $\mathrm{kcal} \cdot \mathrm{mol}^{-1}$; (iv) the reactivity among B-TACs decreases in the order $p d r(\mathbf{1})>p m r(\mathbf{2})>z w$ $(3,4)$, while that among L-TACs decreases in the order $c b\left(5,6^{\prime \prime}\right)>p m r\left(6^{\prime}\right)>z w(7,8)$, in agreement with the expected trend; (v) while B-TACs are meta regioselective, excluding 
carbenoid nitrile ylide 5, L-TACs are ortho regioselective. Consequently, considering the B-TAC/L-TAC pairs, a change in the meta/ortho regioselectivity is observed; (vi) the regioselectivity of these 32CA reactions ranges from 0.8 (TSo-6 $\left.{ }^{\prime \prime}\right)$ to $6.7\left(\right.$ TSo- $\left.6^{\prime}\right) \mathrm{kcal} \cdot \mathrm{mol}^{-1}$, with the reactions of B-TAC $\mathbf{4}$ and L-TACs $\mathbf{6}^{\prime}$ and $\mathbf{8}$ being completely regioselective; (vii) except for L-TAC 7, these 32CA reactions can be considered irreversible as they are exothermic by more than $31 \mathrm{kcal} \cdot \mathrm{mol}^{-1}$ (CAo-6'); (viii) except in the reactions of L-TACs nitrile imine $6^{\prime \prime}$ and nitrile oxide 8 , the regioselectivity is correctly predicted by the analysis of the Parr functions; (ix) the relative energies of the 32CA reactions of L-TACs 5-8 are between $7.8\left(6^{\prime \prime}\right)$ and $13.9(8) \mathrm{kcal} \cdot \mathrm{mol}^{-1}$ higher than those of the B-TAC partners 1-4, indicating, again, the higher reactivity of the latter; and finally, $(\mathrm{x})$ the activation energies involving electrophilic DCE 10 are found between 2.0 (TSo-8) and 10.1 (TSo-6') $\mathrm{kcal} \cdot \mathrm{mol}^{-1}$ lower than those involving ethylene 9 . In addition, the higher reactivity of B-TACs over L-TACs is enhanced in polar reactions.

The gas-phase geometries of the ortho TSs are displayed in Figure 5, while those of the meta TSs are shown in Figure 6. The following conclusions can be drawn from the geometrical parameters: (i) the asynchronicity ranges from 0.20 (TSo-5) to 0.81 (TSm-3) $\AA$; (ii) in general, the ortho TSo-n associated with the B-TACs are more asynchronous than the meta TSm-n ones, while the meta TSm-n associated with the L-TACs are more asynchronous than the ortho TSo-n ones. Therefore, a change in the asynchronicity is found between B-TACs and L-TACs, in agreement with the energy results; (iii) at the fifteen TSs, the $\mathrm{Z3}(\mathrm{X} 1)-\mathrm{C} 4$ distance involving the most electrophilic center of DCE 10 is shorter than the $\mathrm{X} 1$ (Z3) $-\mathrm{C} 5$ one [36]; (iv) the more favorable regioisomeric TSs of these 32CA reactions are much more asynchronous, by between 0.26 (nitrile oxide 2) and 0.80 (azimine 8 ) $\AA$, than those involving ethylene $\mathbf{9}$, due to the high polar character of the former (see later).

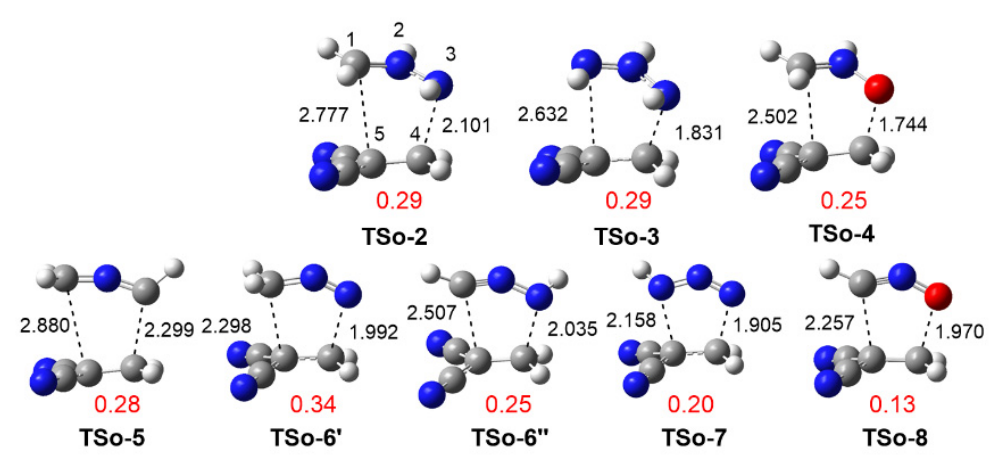

Figure 5. MPWB1K/6-311G $(\mathrm{d}, \mathrm{p})$ gas phase optimized geometries of the TSs associated with the ortho regiosimeric path of the 32CA reactions between TACs 1-8 and DCE 10. Distances are given in angstroms, $\AA$, while the GEDT values computed at the TAC framework, in red, are given in number of electrons, e.
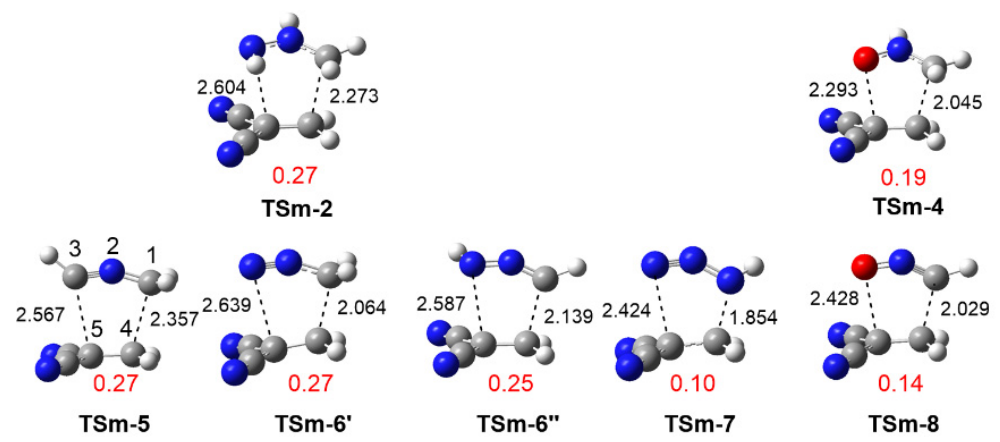

Figure 6. MPWB1K/6-311G $(\mathrm{d}, \mathrm{p})$ gas-phase optimized geometries of the TSs associated with the meta regioisomeric path of the 32CA reactions between TACs 1-4,6,7 and DCE 10. Distances are given in angstroms, $\AA$, while the GEDT values computed at the TAC framework, in red, are given in number of electrons, e. 
The GEDT taking place from the nucleophilic TAC framework to the electrophilic DCE one ranges from 0.10 (TSm-7) to 0.34 (TSm-6 $\mathbf{6}^{\prime}$ ) e (see Figures 5 and 6). Some appealing conclusions arise from these values: (i) except for nitrile oxide 8, the rest of the 32CA reactions have a high polar character, in agreement with the moderate to strong nucleophilicity of these TACs, and the strong electrophilicity of DCE 10 (see Section 3.1); (ii) the direction of the flux of the electron density at the TSs classifies these 32CA reactions as FEDF, in clear agreement with the analysis of the CDFT indices; (iii) these values are by between 0.08 (nitrone 4) and 0.28 (azimine 3) e higher than those associated with the TSs of the non-polar 32CA reactions with ethylene 9; (iv) in general, the meta and ortho TSs display similar GEDT values. Only in the reactions of diazomethane $\mathbf{6}^{\prime}$ and azide $\mathbf{7}$, the GEDT is 0.07 and 0.10 e higher along the preferred ortho path; and finally, (v) there is no clear trend between the GEDT values and the electronic structure of TACs.

\section{Conclusions}

The changes in the electronic structure and reactivity of five pairs of B-TACs and L-TACs participating in 32CA reactions have been studied within the MEDT at the MPWB1K/6-311G(d,p) computational level.

ELF of the nine TACs indicates that while the pseudodiradical and pseudoradical structures of B-TACs $\mathbf{1}$ and 2, respectively, change to a carbenoid structure at L-TACs $\mathbf{5}$ and $\mathbf{6}^{\prime \prime}$, TACs 3 and $\mathbf{4}$ keep their zwitterionic structure at L-TACs $\mathbf{7}$ and 8.

Analysis of the CDFT indices indicates that four of them have a strong nucleophilic character, thus participating in polar reactions towards electrophilic ethylenes. In nonsymmetric TACs 2, 4, 5, 6" and 8, the more electronegative center holds the higher nucleophilic $P_{k}^{-}$Parr function.

The activation energies of the non-polar 32CA reactions towards ethylene 9 range from 0.1 to $21.1 \mathrm{kcal} \cdot \mathrm{mol}^{-1}$. In general, L-TACs are less reactive than B-TACs, with the corresponding TSs being in general less asynchronous. These 32CA reactions have low polar character, such as those involving TACs $\mathbf{1}, \mathbf{2}, \mathbf{6}^{\prime}$ and $\mathbf{1 0}$, or even non-polar character in the cases of TACs $3,4,6^{\prime \prime}, 7$ and 8 .

The activation energies of the polar 32CA reactions involving DCE 10 range from 0.5 to $22.0 \mathrm{kcal} \cdot \mathrm{mol}^{-1}$. The activation energies of the $32 \mathrm{CA}$ reactions involving the four strongest nucleophilic TACs are found between 4.3 and $9.1 \mathrm{kcal} \cdot \mathrm{mol}^{-1}$ lower than those involving ethylene 9. In general, B-TACs are more reactive than the L-TAC counterparts as a consequence of the more nucleophilic character of the former. A change in the regioselectivity is found in these polar 32CA reactions; in general, while the reactions of B-TACs are ortho regioselective, those of L-TACs are meta regioselective. The Parr functions perform well in predicting the regioselectivity in seven of the nine polar 32CA reactions.

At all TSs, the shorter distance involves the most electrophilic C4 carbon of DCE 10. A change in the asynchronicity is found between B-TACs and L-TACs.

Except nitrile oxide 8, all 32CA reactions with DCE 10 have a high polar character, being classified as FEDF. Although a clear trend between the GEDT at the TSs and the electronic structure of TACs cannot be established, the polar character enhances even more the higher reactivity of B-TACs over L-TACs.

In this MEDT study, the structural and reactivity differences between B-TACs and L-TACs species participating in 32CA reactions are examined for the first time, showing proof of the higher reactivity of B-TACs vs. L-TACs as well as their different regioselectivity.

Supplementary Materials: The following are available online at https:/ / www.mdpi.com/article / 10.3390/org2030014/s1, Table S1: MPWB1K/6-311G(d,p) gas phase total electronic energies of the stationary points involved in the 32CA reactions of TACs 1-8 with ethylene 9, Table S2: MPWB1K/6$311 \mathrm{G}(\mathrm{d}, \mathrm{p})$ gas phase total electronic energies of the stationary points involved in the $32 \mathrm{CA}$ reactions of TACs 1-8 with DCE 10, and Cartesian coordinates of the MPWB1K/6-311G $(\mathrm{d}, \mathrm{p})$ gas phase optimized geometries of the TSs involved in the 32CA reactions of TACs 1-8 with ethylene 9 and DCE 10. 
Author Contributions: M.R.-G. headed the subject, wrote the manuscript and performed calculations; L.R.D. headed the subject, wrote the manuscript and performed calculations; and F.G. performed calculations and wrote the manuscript. All authors have read and agreed to the published version of the manuscript.

Funding: This research was funded by the European Union's Horizon 2020 research and innovation program under the Marie Skłodowska-Curie grant agreement No. 846181 (MRG), and by the Ministerio de Ciencias, Innovación y Universidades of the Spanish Government, project PID2019110776GB00 (AEI/FEDER, UE).

Institutional Review Board Statement: Not applicable.

Informed Consent Statement: Not applicable.

Data Availability Statement: Not applicable.

Acknowledgments: This work has been supported by the European Union's Horizon 2020 research and innovation program under the Marie Skłodowska-Curie grant agreement No. 846181 (MRG). This work has also received funding from the Ministerio de Ciencias, Innovación y Universidades of the Spanish Government, project PID2019-110776GB00 (AEI/FEDER, UE).

Conflicts of Interest: The authors declare no conflict of interest.

\section{References}

1. Moss, G.P.; Smith, P.A.S.; Tavernier, D. Glossary of class names of organic compounds and reactivity intermediates based on structure. Pure Appl. Chem. 1995, 67, 1307. [CrossRef]

2. Carruthers, W. Cycloaddition Reactions in Organic Synthesis; Pergamon: Oxford, UK, 1990.

3. Padwa, A. 1,3-Dipolar Cycloaddition Chemistry; Wileynterscience: New York, NY, USA, 1984; Volume 1-2.

4. Domingo, L.R. Molecular electron density theory: A modern view of reactivity in organic chemistry. Molecules 2016, 21, 1319. [CrossRef]

5. Ríos-Gutiérrez, M.; Domingo, L.R. Unravelling the mysteries of the [3 + 2] cycloaddition reactions. Eur. J. Org. Chem. 2019, 267-282. [CrossRef]

6. Domingo, L.R.; Sáez, J.A. Understanding the mechanism of polar Diels-Alder reactions. Org. Biomol. Chem. 2009, 7, 3576-3583. [CrossRef]

7. Domingo, L.R. A new C-C bond formation model based on the quantum chemical topology of electron density. RSC Adv. 2014, 4, 32415-32428. [CrossRef]

8. Domingo, L.R.; Ríos-Gutiérrez, M.; Pérez, P. How does the global electron density transfer diminish activation energies in polar cycloaddition reactions? A Molecular Electron Density Theory study. Tetrahedron 2017, 73, 1718-1724. [CrossRef]

9. Domingo, L.R.; Ríos-Gutiérrez, M.; Pérez, P. A Molecular Electron Density Theory Study of the Reactivity of Tetrazines in Aza-Diels-Alder Reactions. RSC Adv. 2020, 10, 15394-15405. [CrossRef]

10. Domingo, L.R.; Kula, K.; Ríos-Gutiérrez, M. Unveiling the Reactivity of Cyclic Azomethine Ylides in [3 + 2] Cycloaddition Reactions within the Molecular Electron Density Theory. Eur. J. Org. Chem. 2020, 5938-5948. [CrossRef]

11. Houk, K.N.; González, J.; Li, Y. Pericyclic reaction transition states: Passions and punctilios, 1935-1995. Acc. Chem. Res. 1995, 28, 81-90. [CrossRef]

12. Sustmann, R.; Trill, H. Substituent Effects in 1,3-Dipolar Cycloadditions of Phenyl Azid. Angew. Chem. Int. Ed. Engl. 1972, 11, 838-840. [CrossRef]

13. Domingo, L.R.; Mar Ríos-Gutiérrez, M.; Silvi, B.; Pérez, P. The Mysticism of Pericyclic Reactions: A Contemporary Rationalisation of Organic Reactivity Based on Electron Density Analysis. Eur. J. Org. Chem. 2018, 2018, 1107-1120.

14. Zhao, Y.; Truhlar, D.G. Hybrid Meta Density Functional Theory Methods for Thermochemistry, Thermochemical Kinetics, and Noncovalent Interactions: The MPW1B95 and MPWB1K Models and Comparative Assessments for Hydrogen Bonding and van der Waals Interactions. J. Phys. Chem. A 2004, 108, 6908-6918. [CrossRef]

15. Hehre, M.J.; Radom, L.; Schleyer, P.v.R.; Pople, J. Ab Initio Molecular Orbital Theory; Wiley: New York, NY, USA, 1986.

16. Schlegel, H.B. Optimization of equilibrium geometries and transition structures. J. Comput. Chem. 1982, 3, 214-218. [CrossRef]

17. Schlegel, H.B. Modern Electronic Structure Theory; Yarkony, D.R., Ed.; World Scientific Publishing: Singapore, 1994.

18. Fukui, K. Formulation of the reaction coordinate. J. Phys. Chem. 1970, 74, 4161-4163. [CrossRef]

19. González, C.; Schlegel, H.B. Reaction path following in mass-weighted internal coordinates. J. Phys. Chem. 1990, 94, 5523-5527. [CrossRef]

20. González, C.; Schlegel, H.B. Improved algorithms for reaction path following: Higher-order implicit algorithms. J. Chem. Phys. 1991, 95, 5853-5860. [CrossRef]

21. Becke, A.D.; Edgecombe, K.E. A simple measure of electron localization in atomic and molecular-systems. J. Chem. Phys. 1990, 92, 5397-5403. [CrossRef]

22. Reed, A.E.; Weinstock, R.B.; Weinhold, F. Natural population analysis. J. Chem. Phys. 1985, 83, 735-746. [CrossRef] 
23. Reed, A.E.; Curtiss, L.A.; Weinhold, F. Intermolecular interactions from a natural bond orbital, donor-acceptor viewpoint. Chem. Rev. 1988, 88, 899-926. [CrossRef]

24. Parr, R.G.; Yang, W. Density Functional Theory of Atoms and Molecules; Oxford University Press: New York, NY, USA, 1989.

25. Domingo, L.R.; Ríos-Gutiérrez, M.; Pérez, P. Applications of the conceptual density functional indices to organic chemistry reactivity. Molecules 2016, 21, 748. [CrossRef]

26. Parr, R.G.; Szentpaly, L.V.; Liu, S. Electrophilicity index. J. Am. Chem. Soc. 1999, 121, 1922-1924. [CrossRef]

27. Parr, R.G.; Pearson, R.G. Absolute hardness: Companion parameter to absolute electronegativity. J. Am. Chem. Soc. 1983, 105, 7512-7516. [CrossRef]

28. Domingo, L.R.; Chamorro, E.; Pérez, P. Understanding the reactivity of captodative ethylenes in polar cycloaddition reactions. A theoretical study. J. Org. Chem. 2008, 73, 4615-4624. [CrossRef] [PubMed]

29. Kohn, W.; Sham, L.J. Self-consistent equations including exchange and correlation effects. Phys. Rev. 1965, 140, 1133-1138. [CrossRef]

30. Domingo, L.R.; Pérez, P.; Sáez, J.A. Understanding the local reactivity in polar organic reactions through electrophilic and nucleophilic Parr functions. RSC Adv. 2013, 3, 1486-1494. [CrossRef]

31. Frisch, M.J.; Trucks, G.W.; Schlegel, H.B.; Scuseria, G.E.; Robb, M.A.; Cheeseman, J.R.; Scalmani, G.; Barone, V.; Petersson, G.A.; Fox, D.J.; et al. (Eds.) Gaussian 16; Revision, A.03; Gaussian, Inc.: Wallingford, CT, USA, 2016.

32. Noury, S.; Krokidis, X.; Fuster, F.; Silvi, B. Computational tools for the electron localization function topological analysis. Comput. Chem. 1999, 23, 597-604. [CrossRef]

33. Gauss View; Version 6.0; Dennington, R.; Keith, T.A.; Millam, J.M. (Eds.) Semichem Inc.: Shawnee Mission, KS, USA, 2016.

34. Huisgen, R. 1,3-Dipolar Cycloadditions. Proc. Chem. Soc. 1961, 2, 357.

35. Chamorro, E.; Duque-Noreña, M.; Gutierrez-Sánchez, N.; Rincón, E.; Domingo, L.R. A close look to the oxaphosphetane formation along the Wittig reaction: A [2 + 2] cycloaddition? J. Org. Chem. 2020, 85, 6675-6686. [CrossRef] [PubMed]

36. Aurell, M.J.; Domingo, L.R.; Pérez, P.; Contreras, R. A theoretical study on the regioselectivity of 1,3-dipolar cycloadditions using DFT-based reactivity indexes. Tetrahedron 2004, 60, 11503-11509. [CrossRef]

37. Hammond, G.S. A Correlation of Reaction Rates. J. Am. Chem. Soc. 1955, 77, 334-338. [CrossRef] 